\title{
Person-centered risk assessment framework: assessing and managing risk in older adults living with dementia
}

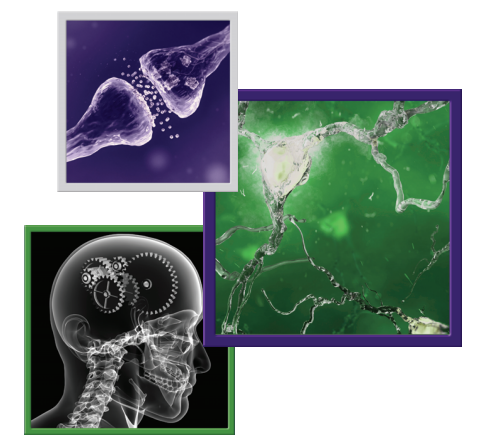

\author{
Linda Lee*,1,2, Loretta M Hillier ${ }^{3}$, Stephanie K Lu ${ }^{1}$, Sharon D Martin ${ }^{1}$, Sarah Pritchard ${ }^{1}$, \\ Jennifer Janzen ${ }^{1} \&$ Karen Slonim ${ }^{1}$ \\ ${ }^{1}$ Center for Family Medicine Family Health Team, McMaster University, 10 B Victoria Street South, Kitchener, Ontario N2G 1C5, \\ Canada \\ ${ }^{2}$ Faculty of Medicine, Department of Family Medicine, McMaster University, 10 B Victoria Street South, Kitchener, Ontario N2G \\ 1C5, Canada \\ ${ }^{3}$ Geriatric Education \& Research in Aging Sciences (GERAS) Centre, St Peter's Hospital, 88 Maplewood Ave, Hamilton, Ontario L8M \\ 1W9, Canada \\ *Author for correspondence: Tel.: +1 519783 0023; Fax: +1 519783 0033; lee.linda.Iw@gmail.com
}

\section{Practice points}

- Persons living with dementia are at increased risk for motor vehicle accidents, getting lost, medication errors and other adverse events.

- There is a critical need for feasible, effective ways of proactively assessing and managing risks associated with dementia, with the aim of averting crises, and unnecessary suffering and costly healthcare service utilization.

- The person-centered risk assessment framework (PCRAF) is a person-centered risk enablement framework that is focused on physical risks, as well as the unintended emotional, psychological and spiritual harm of taking away activities that are meaningful and contribute to quality of life. The PCRAF allows persons with dementia to contribute as much as possible to decisions about high risk activities and alternate solutions to meeting underlying psychosocial needs.

- This study assessed healthcare providers', persons with dementia, and care partners' perceptions of the PCRAF as well as the feasibility of using this framework in Primary Care Collaborative Memory Clinics.

- The PCRAF was well received; use of the PCRAF was sometimes challenging in the context of busy clinic assessments and when the person with dementia had limited insight into potential safety risks.

- Proactive assessment and management of risks may be feasibly conducted within primary care when appropriate to ensure that the care delivered is respectful of individual needs and preferences.

Aim: This study pilot-tested the person-centered risk assessment framework (PCRAF), a framework for managing risk among persons living with dementia (PLWD) in primary care. Methods: Healthcare providers $(\mathrm{N}=7)$ piloting the PCRAF completed a survey, rating their satisfaction with the tool, and an interview to gather their perceptions of the PCRAF. PLWD and care partners $(\mathrm{N}=12)$ completed a survey, rating their satisfaction with safety planning. Results: Care providers were very satisfied with the tool; however, patient or care partner inability to perceive or understand safety risks was a challenge. Use of the PCRAF was perceived as an opportunity to empower self-management, gather PLWD and care partner perspectives, reduce burden for care partners and increase understanding of potential risks. Patients and care partners were very satisfied with the way in which they were included in the risk discussion. Conclusion: The PCRAF is a promising new tool to reduce risks associated with dementia.

First draft submitted: 10 August 2018; Accepted for publication: 22 October 2018; Published online: 14 January 2019

Keywords: caregiving $\bullet$ dementia $\bullet$ memory clinics $\bullet$ primary care $\bullet$ risk assessment

With the aging Canadian population, increasing prevalence of older adults living in the community with dementia and increasing costs of dementia care [1], the healthcare system will be challenged to develop solutions to allow persons suffering from dementia to remain living in the community safely, for as long as possible, and with the best quality of life. Persons living with dementia (PLWD) are at increased risk for motor vehicle accidents, getting 
lost, medical errors and other adverse events that contribute to early institutionalization, and high healthcare utilization [2-4].

Supporting safe longer-term community living among PLWD requires a major shift in approach to care. Traditionally the management of risk has been reactive, occurring after major crises events result in emergency department visits and hospitalizations [5,6]. There is a critical need for feasible, effective ways of proactively assessing and managing risks associated with living with dementia with the aim of averting these crisis events, resulting in avoidance of unnecessary suffering and costly acute care hospital utilization and institutionalization. Importantly, there is need for a person-centered positive risk management framework that recognizes the physical risks and harms of activities made riskier due to memory loss but is balanced by the potential emotional, psychological and spiritual harms associated with loss of activities that are meaningful to that person and contribute to quality of life [7]. Person-centered care is now considered the gold standard of geriatric care and includes essential elements such as the development and ongoing review of individualized, goal-oriented care plans based on person's preferences within an interprofessional team-based approach to care [8]. Risk enablement, involving the balancing of positive benefits from taking risks against the negative effects of attempting to avoid risk altogether, facilitates the development of systems for enabling and managing risks and allows PLWD to retain as much control over their lives as possible; this tailored approach acknowledges that dementia affects different people in different ways and identifies risky situations for individuals rather than viewing every person with dementia as being at equal risk [9].

Although several risk assessment tools are available for use with older adults, such as the Older Americans Resources and Services (OARS) [10] and Identification of Seniors at Risk (ISAR) [11], these tools are not focused on the experience of those living with dementia, are clinically focused on functional status, are not specific to primary care, and assume a fundamentally different approach to risk then a positive risk management approach. Moreover, the reliability and validity of these measures have been questioned, making them generally unsuitable for a community-dwelling person with dementia $[12,13]$. To our knowledge, there are no dementia specific personcentered risk assessment tools for use in the context of Canadian primary care practice.

The Center for Family Medicine (CFFM) Family Health Team Primary Care Collaborative Memory Clinic (PCCMC) developed and pilot tested a person-centered 'risk enablement' framework based on available research evidence and best practices [7,9,14]. In Ontario, Canada, PCCMCs are family physician-led, interprofessional teams within primary care that assess and manage dementia within a shared care approach [15-17]. Consistent with ideal models of chronic disease management [18], the PCCMC model stratifies patients based on risk of poor outcomes and tailors intensity of interventions accordingly, with the majority of dementia care being maintained at the primary care level and only those at highest risk, typically $10 \%$ or less, referred for specialist level care $[15,17,19]$. In this way, the PCCMC model builds capacity within specialist care and primary care to better address the growing need for comprehensive coordinated dementia care with limited available specialist resources. Persons with memory concerns are referred by their family physician to the PCCMC where they and their care partner complete a comprehensive that includes a thorough history, medication review to identify potentially reversible causes, and use of standardized tools to assess cognitive and functional status, depression, care partner burden and assessment of risks including driving safety concerns. All team members work within a synchronous and collaborative approach to identify problematic issues and develop comprehensive and proactive care plans. Detailed descriptions of this care model, training program and continuing education, and impacts related to access to community services, referring physicians and the health system are presented elsewhere [15-17,19-25]. The PCCMC person-centered risk assessment framework (PCRAF) is a proactive approach that allows older adults living with dementia to retain as much control over their lives as possible, identifying risky situations and developing systems to manage risk. A person-centered approach to care, considered by many to be the gold standard for healthcare for older adults, emphasizes individual preferences, goals and values as well as choice and autonomy and aims to improve healthcare safety, quality, care coordination and quality of life for older adults $[8,26]$. The PCRAF contributes to person-centered care with the development of an individualized goal-oriented care plan based on the person's preferences and ongoing review of the person's goals and care plan, and care supported by an interprofessional team in which the person is an integral member. The PCRAF reflects a change in approach from considering 'risk' in absolute terms to better understanding vulnerability within the context of the situations that make a particular risky, and if possible managing potential risks and harms using an approach that places the person at the center of the decision-making to make the situation less risky [7]. And if circumstances cannot be mitigated to reduce substantive risk, the next task is to determine the underlying psychosocial needs that are met by that particular activity and to work toward finding new meaningful activities that might fulfil those underlying psychosocial needs [9]. 
This paper describes the PCRAF and presents the results of a pilot study to study the feasibility of its use in PCCMCs and Adult Day Programs and to describe user and care recipient reactions to the PCRAF.

\section{Person-centered risk assessment framework}

The PCRAF aims to engage PLWD in risk assessment and creating a positive person-centered care plan. Risky situations include circumstances that increase the risk of falls, financial abuse, physical abuse, alcohol excess, unsafe driving and medication mismanagement or inability to manage selfcare resulting in destabilization of co-existing chronic complex conditions that drive emergency department visits, lengthy hospitalizations with alternate level of care designations and premature institutionalization. The PCRAF assists healthcare providers to identify risky situations based on interviews with the PLWD and their care partners and, through shared understanding and drawing on knowledge of past experiences of the individual, develop a plan for balancing the physical components of risk with the psychosocial aspects of risk, such as the effects on well-being or self-identity whether persons are unable to engage in activities that are important to them. As part of this risk assessment process, PCCMC team members will obtain input from the PLWD and care partners and consider the extent to which a particular activity contributes to the person's quality of life and, if taken away, will reduce quality of life [9,27]. Resulting plans may include the development of a supportive physical and social environment that can enable continued participation in enjoyable activities with reduced risk of harm, or it may include replacing the original high-risk activity with new meaningful activities that meet the basic underlying psychosocial need provided by the original activity. These include the need for love (feeling unconditionally accepted), comfort (feelings of closeness to others), identity (knowing your story and who you are), occupation (activities with personal significance), inclusion (having a distinct place in a group), attachment (specific emotional bonds with people, pets, places, objects), or environment (sense of safety and security) [9]. Although shared agreement may not always be possible, the tool promotes a shared understanding of the viewpoints of all who are affected by the decisions involving risk and emphasizes the importance of identifying meaningful activities for PLWD that meet fundamental psychological and social needs that contribute to quality of life. This approach provides an opportunity for the person with dementia to contribute as much as possible to decisions about high risk activities and alternate solutions to meet underlying psychological needs.

The PCRAF consists of two components: Person-centered risk assessment and My Plan, which creates a plan or strategy for managing risk based on the activities associated with each risk (e.g., the risk is fire, the activity is cooking). Intervention planning focuses on understanding the underlying psychological or social need associated with the risky activity ('I value this activity because...', ensuring the person with dementia understands the risks ('The risks associated with this activity are...') and determining whether these needs can be met in other ways (I will use these strategies to reduce my risk...). The PCRAF is presented in Figure 1. The assessment is typically completed the PCCMC social worker, occupational therapist (OT), or the Alzheimer Society team member, or whichever team member completed the caregiver interview portion of the regular PCCMC assessment.

The PCRAF was developed, pilot tested and further refined within the CFFM PCCMC. Preliminary informal feedback was positive with respect to feasibility and acceptability from care providers administering this tool and care recipient perspectives. This study aimed to further pilot test the tool in other PCCMCs and community settings in order to determine the feasibility of more widespread dissemination.

\section{Methods}

This study used a mixed methods (quantitative and qualitative) approach. Approval was granted from the Hamilton Integrated Research Ethics Board, McMaster University.

\section{Participants}

Seven healthcare providers (three OT, two social workers and two Alzheimer Society representatives, one an OT, the other a social worker) working in 13 PCCMCs were tasked with identifying individuals assessed in the PCCMC who were potentially living at risk and could benefit from a risk assessment. PLWD were deemed eligible to complete the PCRAF based on the care providers' clinical judgement that the person had the capacity to understand the intent of the tool. An Alzheimer Society Adult Day Program in Kitchener, Ontario, was recruited to test the PCRAF with a purposeful sample of their clients who may potentially be living risk. PLWD and their care partners who had completed the PCRAF in their PCCMC or Adult Day Program were also invited to participate in this study. The PCRAF was piloted for 6-month period from April to September 2017. 
1. Identify the activity/circumstance associated with risk

> E.g., wandering/exit seeking, getting lost, falls, driving, cooking, abuse/neglect, medication management, handling of firearms, suicidal ideation, etc.

2. Identify the level of risk perceived by:

$>$ The caregiver: high/intermediate/low risk

$>$ The patient: high/intermediate/low risk

If there is discrepancy, which perception seems most accurate? Can there be agreement?

3. Does the activity meet an underlying psychosocial need for the patient*?

i. Love (feeling unconditionally accepted)

ii. Comfort (feelings of closeness to others)

iii. Identity (knowing your story and who you are)

iv. Occupation (activities with personal significance)

v. Inclusion (having a distinct place in a group)

vi. Attachment (specific emotional bonds with people, pets, places, objects)

vii. Environment (sense of safety and security)

4. Can the activity be made less risky?

$>$ If so, complete "My Plan" with patient and caregiver together

$>$ If not, determine an alternate activity that can meet the underlying psychosocial need

Activity:

I value this activity because...

The risks associated with this activity are...

$>$ Consider linking to community resources (e.g., Safely home, H\&CC, Alzheimer society, Behaviour supports ontario)

$>$ Contact authorities as appropriate (e.g., Elder Abuse team, fire prevention officer)

$>$ Ensure regular review of My Plan

Figure 1. Person-centered risk assessment framework: laminated point-of-practice card (double-sided).

Note: The Primary Care Collaborative Memory Clinics have recently changed their name to MINT (Multispecialty Interprofessional Team-Based Memory Clinic)

(c) 2018 L Lee, S Dillon-Martin, S Pritchard. Center for Family Medicine Memory Clinic. This tool is a conceptual aid for healthcare providers. It is not a substitute for physician's diagnosis and treatment and it is not medical advice. Use at your own risk. All rights reserved. This tool may be reproduced and disseminated, 'as is' without any modification, adaptions or translations, for personal, educational, nonprofit, noncommercial purposes provided acknowledgment of the authors and the Center for Family Medicine Memory Clinic is made. This document may not be modified, adapted, translated, be made for resale or for any other commercial purpose whatsoever without prior permission in writing from the Center for Family Medicine Memory Clinic. 


\section{Measures}

PCCMC care providers and Adult Day Program staff were asked to track the total number of patients assessed in the PCCMC during the pilot-testing time period, and of these, the number of patients deemed eligible to complete the PCRAF.

Following the pilot testing period, care providers $(\mathrm{N}=7)$ were invited to complete an on-line survey to rate their satisfaction (five-point scale: 1 = not at all satisfied, 5 = extremely satisfied) with the tool overall, ease of use, acceptability for patients and feasibility of use, and to rate their level of agreement (five-point scale: strongly disagree to strongly agree) with various statements about the PCRAF as related to its inclusion of patient perspectives, practice relevance and reflection of real risks that exist. In addition, care providers were invited to participate in individual telephone interview to gather information about their perceptions of the PCRAF in terms of its strengths, challenges and associated benefits.

After having completed the PCRAF, in either a PCCMC or Adult Day Program, PLWD and care partners were invited to complete a paper-based survey in which they were asked to rate their satisfaction (five-point scale: $1=$ not at all satisfied, $5=$ extremely satisfied) with the risk discussion and whether they thought the plan would improve safety risk and their intentions to follow the plan. An open-ended response question was used to solicit their perceptions of anticipated benefits associated with the PCRAF.

All of the tools developed for this study were pilot-tested with CFFM clinicians and revised accordingly prior to use in this study.

\section{Data analysis}

Quantitative (survey) data were analyzed using SPSS 25.0 software (IBM Corp, Armonk, NY: IBM Corp) to generate descriptive statistics (frequencies, means, and standard deviations [SD]); content analysis was used for open-ended survey data [28]. All interviews were conducted by one author (LMH) to ensure consistency and digitally recorded and transcribed. Interview transcripts were analyzed using a naturalistic inquiry approach to develop an understanding of care providers and patient experiences within their individual context. Responses to each question were categorized and contrasted to create a summary of responses to identify recurring themes in the data consistent with common practices for qualitative data [29]. Interview analysis was conducted by one research team member $(\mathrm{LMH})$ and then reviewed by a second research team member (SKL), who also reviewed the transcripts to identify and finalize key themes generated by the analysis. Study rigor was further ensured with an audit trail of decisions related to participant recruitment, data collection and analysis, as well as overall feedback from the entire research team on the analysis and study findings and conclusions.

\section{Results}

Across all PCCMCs, a total of 297 patients were assessed during the study time period. Of these patients, 43 (14.5\%) were deemed appropriate to complete the PCRAF, and of these patients, the PCRAF was completed with 23 (53.5\%); this represents $14.5 \%$ (23/297) of all patients assessed during the study time period and 53.5\% (23/43) of all patients deemed appropriate for the PCRAF. Within the Adult Day Program, the PCRAF was completed with eight individuals. Across both settings, the PCRAF was completed with 31 individuals.

\section{Health care provider survey}

The survey was completed by six care providers (four social workers, two OT); two reported never having had an opportunity to use the tool. Overall, those who used the PCRAF were very satisfied with the tool overall (mean $=3.5, \mathrm{SD}=0.58)$ and moderately satisfied with ease of use (mean $=2.8, \mathrm{SD}=0.58)$, acceptability for patients (mean $=2.3, S D=58)$, and feasibility for use (mean $=2.5, \mathrm{SD}=1.3$ ) (Figure 2$)$. All agreed that the PCRAF facilitates the development of a person-centered risk management plan; almost all (75\%) agreed that it was relevant to their practice and accurately reflected existing risks.

\section{Health care provider interviews}

Interviews were conducted with six care providers (86\% response rate). Four themes were generated related to key strengths of the PCRAF; interview participants described the PCRAF as: comprehensive; promoting independence and a proactive approach to dementia; empowering patients to be involved in care planning; and, a good communication tool to increase clinic team awareness of existing risks. These strengths are summarized in Table 1 with illustrative quotes. 


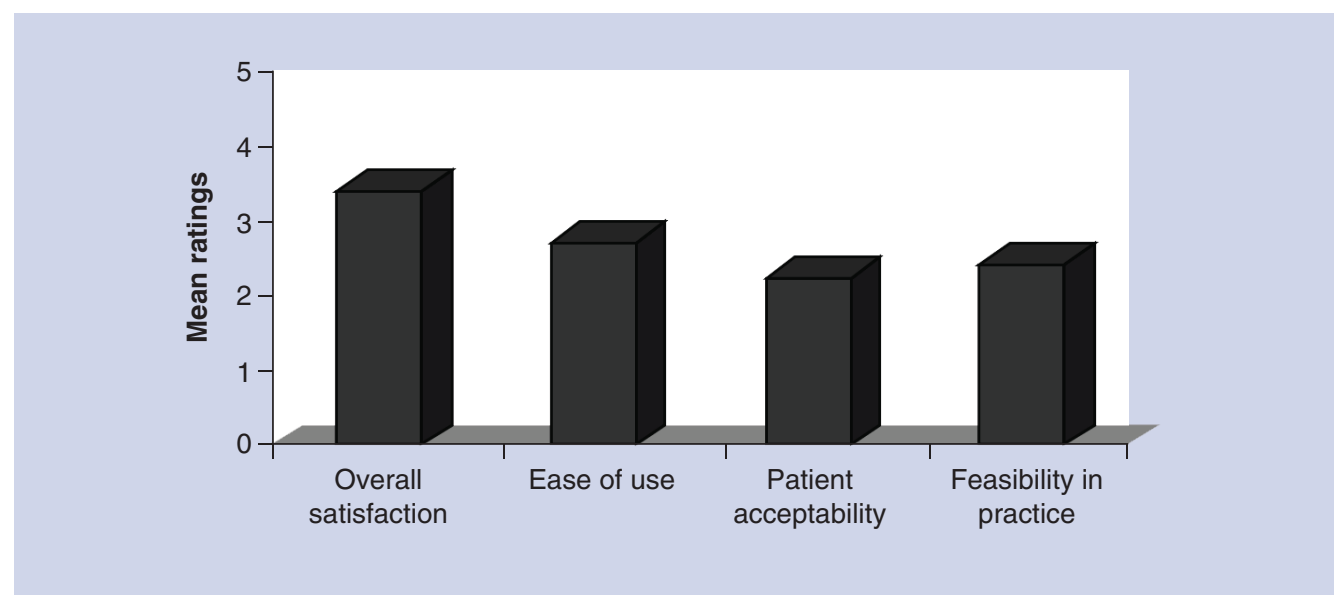

Figure 2. Healthcare providers' ratings of various aspects of the person-centered risk assessment framework $(\mathrm{N}=4)$. Ratings 5-point scale: 1 = not at all satisfied; 5 = extremely satisfied.

\begin{tabular}{|c|c|c|}
\hline Themes & & Illustrative quotes \\
\hline \multirow[t]{4}{*}{ Strengths } & Comprehensiveness & "I like the fact that obviously it lists pretty much every risk that you can think of" [HCP1] \\
\hline & $\begin{array}{l}\text { Promotion of independence and a proactive } \\
\text { approach to dementia }\end{array}$ & $\begin{array}{l}\text { "The fact that it is giving somebody with a dementia the opportunity to hopefully be } \\
\text { more independent and proactive when it comes to situations where their caregiver } \\
\text { might be a little bit uncomfortable with the risk of putting themselves in, you know, } \\
\text { put the power back in their hands to change it" [HCP1] }\end{array}$ \\
\hline & $\begin{array}{l}\text { Empowerment of patients to be involved in care } \\
\text { planning }\end{array}$ & $\begin{array}{l}\text { "I think it's a good tool for sure. Definitely, and I like the piece of if you have somebody } \\
\text { who is insightful into their diagnosis, building in that my plan piece, its huge" [HCP4] }\end{array}$ \\
\hline & $\begin{array}{l}\text { Communication tool to increase awareness of } \\
\text { existing risks among clinic team members }\end{array}$ & $\begin{array}{l}\text { "What I like about it is that if you do something like this then everybody is sort of on } \\
\text { the same board. Everybody knows what the risk is. All the team members can be } \\
\text { involved, like the GP or the physicians that we work with are aware of our concerns } \\
{[\ldots] \text { I think it's good in that it's a visual representation" [HCP6] }}\end{array}$ \\
\hline \multirow[t]{4}{*}{ Challenges } & $\begin{array}{l}\text { Assessment visit is not an optimal time to } \\
\text { administer the PCRAF }\end{array}$ & $\begin{array}{l}\text { "Because of the nature of how it's [assessment results] received, the insight into them } \\
\text { even recognizing that they have memory loss [...] It doesn't feel right and it feels like } \\
\text { it would be more about while at the same time we're trying to advocate for the voice } \\
\text { of the person, our intentions are pure, but we're not meeting them where they are. } \\
\text { They're ticked off, they're devastated, and the caregiver is crying because how am I } \\
\text { going to do it. Is that the best place for that [PCRAF] and the best time?" [HCP2] }\end{array}$ \\
\hline & $\begin{array}{l}\text { The PCRAF is not well integrated into the } \\
\text { assessment process }\end{array}$ & $\begin{array}{l}\text { "I think I found it hard for me to use it because it's a lot of forms that we already go } \\
\text { through, maybe if it could be integrated better" [HCP3] }\end{array}$ \\
\hline & $\begin{array}{l}\text { The PCRAF may duplicate risk assessments } \\
\text { conducted with caregivers and in-home functional } \\
\text { assessments }\end{array}$ & $\begin{array}{l}\text { "Inherently when I go out to do a home visit right, again as an OT you're sort of always } \\
\text { addressing what are the potential risks [...] So I'm inherently always sort of doing this } \\
\text { stuff [risk assessment]" [HCP6] }\end{array}$ \\
\hline & Patient and/or caregiver failure to recognize risks & $\begin{array}{l}\text { "A lot of times our caregivers didn't identify that risk [...] in the caregiver interview } \\
\text { they're saying: 'Oh, they're [PWD] great drivers, no issues, no problems'. And then you } \\
\text { get their testing [results] and you're like whoa! That's very risky! So a lot of times my } \\
\text { caregivers are like 'No, its fine'. Then you can't move forward with it. So and that kind } \\
\text { of is the first roadblock and even if the caregivers do identify a risk, I can't move } \\
\text { forward with it unless the patient has identified that risk which is kind of the second } \\
\text { roadblock I deal with. [...] The insight piece is definitely the biggest one [challenge]" } \\
\text { [HCP4] }\end{array}$ \\
\hline
\end{tabular}

PCRAF: Person-centered risk assessment framework.

A number of themes were generated related to challenges to the implementation of the PCRAF: the assessment visit is not an optimal time to administer the PCRAF because patients and care partners are still grappling with diagnosis they were given, limited time available in the already lengthy clinic appointment and the discussion requires a more established relationship with between patients and care providers; the PCRAF is not well integrated into the assessment process; the PCRAF may duplicate questions asked of caregivers in their assessment interviews and in OT-led home visits; and some patients and/or caregivers fail to recognize risks either because of lack of 
Table 2. Impacts associated with the person-centered risk assessment framework as identified by interviewed healthcare professionals.

\begin{tabular}{|c|c|}
\hline Themes & Illustrative quotes \\
\hline $\begin{array}{l}\text { PCRAF's patient-centered approach empowers } \\
\text { and contributes to self-management }\end{array}$ & $\begin{array}{l}\text { "I generally work with the caregiver and I look at it from a caregiver perspective. It's sort of made } \\
\text { me think about the person themselves and what might they be experiencing and letting them take } \\
\text { the lead. So giving the power to them too. So I really like that, it's a shift in focus [...] and looking } \\
\text { also at the person with dementia as being a key player in their own healthcare" [HCPID3] } \\
\text { "It also increases the self-management. I think it's empowering for the patients as well [...] I think it } \\
\text { kind of empowers them because they're participating in it and they come up with the solution so it's } \\
\text { more likely to be successful than the method of going in seeing things and then making } \\
\text { recommendations" [HCP5] }\end{array}$ \\
\hline $\begin{array}{l}\text { PCRAF provides structured opportunities for } \\
\text { patients and caregivers to share their perspectives }\end{array}$ & $\begin{array}{l}\text { "In an intentional way it's very powerful when you hear a person living with dementia talking with } \\
\text { their care partner back and forth, back and forth. 'Why is this important to me'. 'Well I didn't know } \\
\text { it was that important to you'. 'I see this risk'. 'Well I didn't know you saw it that way'. That back and } \\
\text { forth, its wow!" [HCPID2] } \\
\text { "The driving issue, so you know, care givers are saying I don't want to get into a car with him, he's } \\
\text { saying that there's no risk, at least it kind of gives them an opportunity to sort of discuss that, feel } \\
\text { heard" [HCP5] }\end{array}$ \\
\hline $\begin{array}{l}\text { PCRAF reduces the care burden for caregivers by } \\
\text { having the process of identifying and reducing } \\
\text { risk led by someone other than themselves }\end{array}$ & $\begin{array}{l}\text { "They feel they gain so much more insight and they gain so much more genuine I'll call it } \\
\text { compassion or empathy, but maybe more empathy and that allows them to maybe: 'Okay let's take a } \\
\text { deep breath here and realize I don't have to fix everything'. I don't have to be in charge and tell } \\
\text { them [PWD] what to do and when to do it and how to do it [...] the caregiver has an opportunity } \\
\text { here to learn that they have a voice and that maybe we can work together rather than [caregivers] } \\
\text { react to it and start taking away meaningful activities" [HCP2] } \\
\text { "I think it takes some onus off the caregiver too maybe [...] and they're not the one responsible for } \\
\text { trying to you know, initiate things all the time" [HCP3] }\end{array}$ \\
\hline PCRAF identifies and manages risk & $\begin{array}{l}\text { "I think again going back to you know, having us as memory clinic staff looking at risks more often, } \\
\text { like having it in the forefront of our minds through every appointment might help us identify some } \\
\text { of those risks a little bit earlier and hopefully make plans with the care partner and the patient to } \\
\text { maybe reduce some of those" [HCP4] } \\
\text { "Well I think identifying it and addressing it hopefully we minimize the risk and if we minimize the } \\
\text { risk then we minimize like hospitalization, injury" [HCP6] }\end{array}$ \\
\hline $\begin{array}{l}\text { PCRAF assists care partners to have a realistic } \\
\text { understanding of patient's capacity for } \\
\text { independence. }\end{array}$ & $\begin{array}{l}\text { "The other thing I found is that sometimes family members think okay, they have memory issues, } \\
\text { they underestimate how much the patient can still participate in the activities of daily living" [HCP5] } \\
\text { "Sometimes family don't live with them and they are identifying all these risks and then when we go } \\
\text { and see how they're doing at home, they're actually managing quite well" [HCP5] }\end{array}$ \\
\hline
\end{tabular}

insight or denial, thus making it difficult to complete the assessment. These challenges are summarized in Table 1 with illustrative quotes.

A number of themes were identified related to impacts associated with the PCRAF; the PCRAF: empowers and facilitates self-management; provides structured opportunities for patients and care partners to share their perspectives; reduces care burden for care partners; better identifies and manages risk; and, assists care partners to have a realistic understanding of patient's capacity for independence. These themes are summarized in Table 2 with illustrative quotes.

\section{Persons living with dementia \& care partner survey}

Twelve surveys were completed by PLWD $(\mathrm{N}=4)$ and care partners $(\mathrm{N}=8)$; six were completed by individuals assessed in PCCMCs and six assessed in the Adult Day Program. Respondents were very satisfied with: the way in which their opinions were included in the risk discussion (mean $=4.4, \mathrm{SD}=0.51$ ), talking about safety risks with the healthcare provider $($ mean $=4.3, \mathrm{SD}=0.65)$, and the safety risk plan that was developed $(\mathrm{mean}=4.1, \mathrm{SD}=$ 0.99) (Figure 3).

Almost all of the respondents $(5 / 6 ; 83.3 \%)$ reported that they thought the plan that was developed would help to improve safety risk. Similarly, almost all respondents $(5 / 6,83.3 \%)$ indicated that they had intentions to follow plan (2/5 - 'all of it'; 3/5 - 'most of it') and one respondent indicated 'maybe'. The value of the safety plan is reflected in the following comments: 


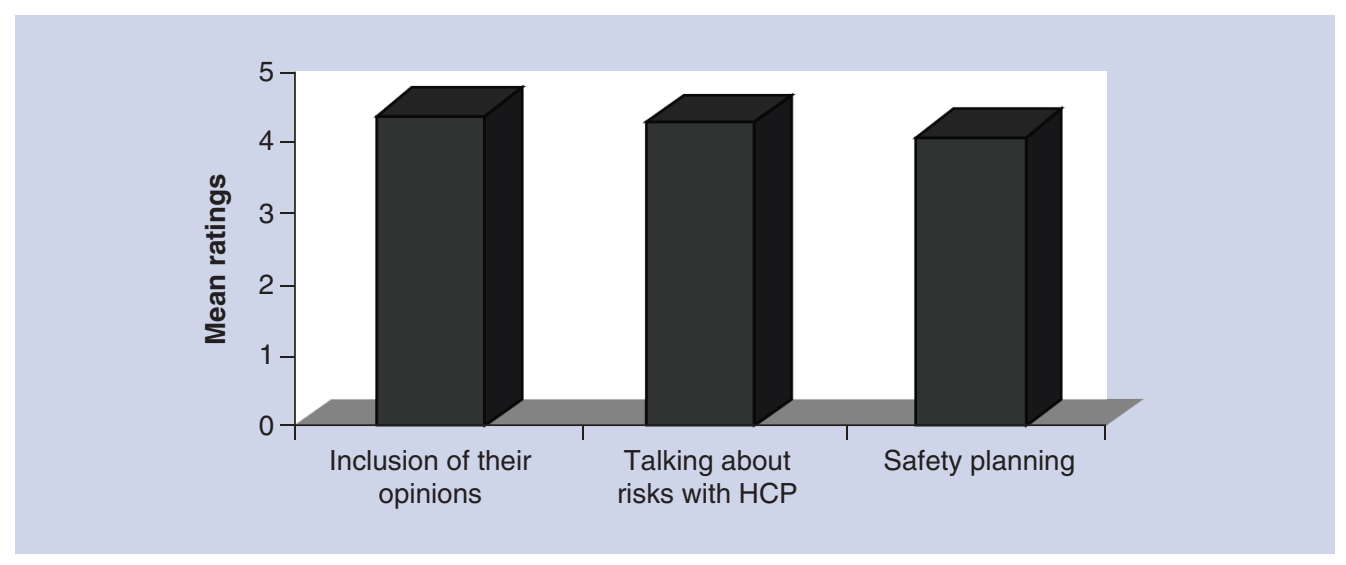

Figure 3. Persons living with dementia and care partner ratings of satisfaction with various aspects of the person-centered risk assessment framework $(\mathrm{N}=12)$.

Ratings 5 -point scale: 1 = not at all satisfied; 5 = extremely satisfied.

\begin{abstract}
"Because my father lives alone and has aphasia, I am often concerned if there was an emergency and his ability to communicate what has occurred. Having Lifeline and red flagging his address with emergency services will help to alleviate stressors for him in having to directly make these calls. " [Care partner]

"It is comprehensive. It makes me face difficult issues and deal with it. It helps me connect with other health providers. "[PLWD]

"This comprehensive care is truly appreciated." [PLWD]
\end{abstract}

\title{
Discussion
}

The PCRAF is a promising new approach for assessing and managing risks associated with living with dementia. In this study, the PCRAF was valued for its comprehensiveness and shared discussions about safety risks and potential solutions incorporating the perspectives of the individual. A tool that is anchored in a person-centered positive risk management framework has the potential to ensure that the recognition of physical risks and harms of activities made riskier due to memory impairment is balanced by the consideration of potential emotional, psychological and spiritual harms of taking away meaningful activities that contribute to quality of life [7]. The development of systems for enabling and managing risk, incorporating a shared understanding of the viewpoints of all who are affected by the decisions involving risk, can allow PLWD to retain as much control over their lives as possible and help them and their care partners to maintain best quality of life living in the community. This study identified some potential important impacts associated with the PCRAF that suggest that there is value in improving processes for implementing this tool and further evaluative studies. A key strength of PCRAF identified in this is study is its person-centered approach.

This study validated the value of the person centered approach to risk; however, it also identified previously unrecognized feasibility issues regarding implementation by PCCMCs that needed to be addressed prior to widespread use. Finding time to complete the tool within the context of the already comprehensive clinic assessment was an identified barrier to its use; to address this, some care providers opted to complete the tool during home visits. Although this was perceived as a viable solution, it does limit the use of the tool to care providers able to offer a home visit. Our team has reviewed the study findings and identified areas for improving implementation, which we will then re-evaluate, consistent with Plan, Do, Study, Act (PDSA) cycles [30]. Incorporating results of feedback from this study, a simple point-of-practice tool will be distributed in the form of a laminated card (Figure 1) to serve as a reminder for care providers of the key concepts to consider when using a person-centered risk assessment approach for addressing unsafe activities. This card will be distributed, along with a presentation on the PCRAF, at an upcoming education event held annually for all memory clinic team members across the province [23]. There is evidence that supporting education with point-of-practice tools can effectively facilitate knowledge transfer to practice [31].

There are several limitations to this study. The sample size for this study in terms of number care provider testing the PCRAF was small, however, was likely sufficient for the purposes of this pilot study. The PCRAF appears most 
ideally suited to persons at the early to moderate stage of dementia when concerns about risky activities emerge, but the person still has capacity to understand the risks identified and able to contribute to the plan to mitigate the risks of that activity. Given the short time frame of the study, and the fact that PCCMCs see persons not only with dementia but also other memory disorders, there was not adequate opportunity to capture a large number of PLWD whose care partners identified a risky activity, but at a stage of dementia not so advanced that the PLWD was still able to meaningfully contribute to a plan of management. Healthcare providers used the PCRAF less than they had anticipated due to lack of opportunity (few patients identified as living at high risk), lack of time within the clinic assessment to explore potential risks, risk being assessed by another community service, or existing risk being identified but already addressed in prior clinic visits. Inability of patients or care partners to perceive or understand safety risks, options available for mitigating these risks and the potential consequences of choosing one option over the other was also identified as a barrier to using the PCRAF.

Despite these limitations, our findings support the value of this tool in identifying and mitigating risks and doing so in a way that involves the perspectives of both patients and care partners and supports caregivers' efforts to keep their family member safe while promoting quality of life. Even when the person lacks this capacity, it is helpful to understand what underlying psychosocial needs that the activity met and to help explore other meaningful activities that might fulfil that need. There is a need to go beyond the physical components of risk to considering the psychosocial aspects of risk, such as the effects on well-being or self-identity if a person is unable to do something that is important for them [9]. The assessment and mitigation of risk is an ongoing process; while risks associated with various activities (e.g., food preparation, shopping, banking, dressing, roaming, use of tools) are explored at each PCCMC visit, use of the PCRAF on a regular basis can support more formal processes to systematically identify and manage risk through a person-centered lens. Consistent use of this tool may serve to increase patient and care partner understanding over time of the potential risks that exist, but most importantly recognizing the PLWD's input being an integral part of the plan of care and respecting the goals, values and preferences of the individual

\section{Conclusion}

The PCRAF is a promising person-centered approach to address the critical need for feasible, effective ways for care providers to proactively assess and manage risks associated with dementia. A tool that is anchored in a personcentered positive risk management framework has the potential to ensure that the recognition of physical risks and harms of activities made riskier due to memory impairment is balanced by the consideration of potential emotional, psychological and spiritual harms of taking away meaningful activities that contribute to quality of life. Proactive assessment and management of risks may be feasibly conducted by care providers to ensure that management recommendations are respectful of individual needs and preferences. With further refinement and testing, an improved PCRAF has the potential to assist PCCMCs to identify and manage risk, incorporating a shared understanding of the viewpoints of all who are affected by the decisions involving risk, and allowing PLWD to retain as much control over their lives as possible and help them and their care partners to maintain best quality of life living in the community.

\section{Acknowledgments}

The authors gratefully acknowledge the assistance of memory clinic team members, Adult Day Program staff, persons with dementia and care partners who participated in this study.

Financial \& competing interests disclosure

This study was supported by a SPARK program grant from the Canadian Centre for Aging and Brain Health Innovation (CC-ABHI). The authors have no other relevant affiliations or financial involvement with any organization or entity with a financial interest in or financial conflict with the subject matter or materials discussed in the manuscript apart from those disclosed.

No writing assistance was utilized in the production of this manuscript.

\section{Ethical conduct}

The authors state that they have obtained appropriate institutional review board approval or have followed the principles outlined in the Declaration of Helsinki for all human or animal experimental investigations. In addition, for investigations involving human subjects, informed consent has been obtained from the participants involved. 


\section{References}

Papers of special note have been highlighted as: $\bullet$ of interest

1 Alzheimer's Society of Canada. Prevalence and monetary costs of dementia in Canada (2016). www.alzheimer.ca/ /media/Files/national/Statistics/PrevalenceandCostsofDementia_EN.pdf

2 Steele C, Rovner B, Chase GA, Folstein M. Psychiatric symptoms and nursing home placement of patients with Alzheimer's disease. Am. J. Psychiatry 147(8), 1049-1051 (1990).

3 Rowe M, Greenblum CA, D’Aoust RF. Missing incidents in community-dwelling people with dementia. Am. J. Nurs. 112(12), 30-35 (2012).

4 Sternberg SA, Wolfson C, Baumgarten M. Undetected dementia in community dwelling older people: the Canadian study of health and aging. J. Am. Geriatr. Soc. 48(11), 1430-1434 (2000).

5 Gaugler JE, Kane RL, Kane RA, Newcomer R. Early community-based service utilization and its effect on institutionalization in dementia caregiving. Gerontologist 45(2), 177-185 (2005).

6 Pratt R, Clare L, Kirchner V. 'It's like a revolving door syndrome': professional perspectives on models of access to services for people with early-stage dementia. Aging Ment. Health 10(1), 55-62 (2006).

7 Clarke C, Mantle R. Using risk management to promote person-centred dementia care. Nurs. Stand. 30(28), 41-46 (2016).

8 American Geriatrics Society Expert Panel on Person-Centred Care. Person-centered care: a definition and essential elements. J. Am. Geriatr. Soc. 64(1), 15-18 (2016).

- Provides a good description of person-centered care.

9 UK Government Department of Health. Nothing ventured, nothing gained': risk guidance for people with dementia. UK Government Department of Health. London, UK (2010).

https://assets.publishing.service.gov.uk/government/uploads/system/uploads/attachment_data/file/215960/dh_121493.pdf

10 Fillenbaum GG. Multidimensional functional assessment of older adults.. The Duke Older Americans Resources and Services Procedures. Laurence Erlbaum, New Jersey, NY, USA (1988).

11 McCusker J, Bellavance F, Cardin S, Trepanier S, Verdon J, Ardman O. Detection of older people at increased risk of adverse health outcomes after an emergency visit: the ISAR screening tool. J. Am. Geriatr. Soc. 47(10), 1229-1237 (1999).

12 Burholt V, Windle G, Ferring D et al. Reliability and validity of the older Americans resources and services (OARS) social resources scale in six European countries. J. Gerontol. B Psychol. Sci. Soc. Sci. 62(6), S371-S379 (2007).

13 Edmans J, Bradshaw L, Gladman JRF et al. The identification of seniors at risk (ISAR) score to predict clinical outcomes and health service costs in older people discharged from UK acute care medical units. Age Ageing 42(6), 747-753 (2013).

14 Wolverson EL, Clarke C, Moniz-Cook ED. Living positively with dementia: a systematic review and synthesis of the qualitative literature. Aging Ment. Health 20(7), 676-699 (2016).

- This review describes the conceptual domains that define positive outcomes for people who live with dementia.

15 Lee L, Hillier LM, Stolee P et al. Enhancing dementia care: a primary care-based memory clinic. J. Am. Geriatr. Soc. 58(11), 2197-2204 (2010).

16 Lee L, Weston WW, Hillier LM. Developing memory clinics in primary care: an evidence-based interprofessional program of continuing professional development. J. Contin. Educ. Health Prof. 33(1), 24-32 (2013).

17 Lee L, Hillier LM, Molnar F, Borrie MJ. Primary care collaborative memory clinics: building capacity for optimized dementia care. Healthc. Q. 19(4), 55-62 (2017).

- Provides a detailed description of the Primary Care Collaborative Memory Clinic care model and its impact on the system of care for persons with dementia.

18 Scott I. Chronic disease management: a primer for physicians. Intern. Med. J. 38(6), 427-437 (2008).

19 Lee L, Hillier LM, McKinnon Wilson J et al. Effect of primary care-based memory clinics on referrals to and wait-time for specialized geriatric services. J. Am. Geriatr. Soc. 66(3), 631-632 (2018).

20 Lee L, Hillier LM, Heckman GA et al. Primary care based memory clinics: expanding capacity for dementia care. Can. J. Aging 33(3), 307-319 (2014).

21 Lee L, Hillier LM, Weston W. Ensuring the success of interprofessional teams: key lessons learned in memory clinics. Can. J. Aging 33(1), 49-59 (2014).

22 Lee L, Kasperski MJ, Weston WW. Building capacity for dementia: training program to develop primary care memory clinics. Can. Fam. Physician 57(7), e249-e252 (2011).

23 Lee L, Hillier LM, Weston WW. 'Booster days': an educational initiative to develop a community of practice of primary care collaborative memory clinics. Gerontol. Geriatr. Educ. 5, 1-16 (2017).

24 Lee L, Hillier LM, Harvey D. Integrating community services into primary care: improving the quality of dementia care. Neurodegener. Dis. Manag. 4(11), 11-21 (2014). 

meeting fundamental psychological needs. Perspect. Public Health 136(2), 99-107 (2016).

28 Prim. Care 21(2), 123-130 (2013).

31 Thomas DC, Johnston B, Dunn Ket al. Continuing medical education, continuing professional development, and knowledge translation: Improving care of older patients by practicing physicians. J. Am. Geriatr. Soc. 54(10), 1610-1618 (2006).

Lee L, Hillier LM. Family physicians' perspectives on memory clinics in primary care. Neurodegener. Dis. Manag. 6(6), 467-478 (2016).

Kogan AC, Wilber K, Mosqueda L. Person-centered care for older adults with chronic conditions and functional impairment: a systematic literature review. J. Am. Geriatr. Soc. 64(1), e1-e7 (2016).

Cavanagh S. Content analysis: concepts, methods, and applications. Nurse Pract. 4(5), 16 (1997).

Patton MQ. Qualitative Evaluation and Research Method. Sage, Thousand Oaks, CA, USA (2002).

Gillam S, Siriwardena AN. Frameworks for improvement: clinical audit, the plan-do-study-act cycle and significant event audit. Qual. 
